\title{
RECOVERY OF SCATTERED DATA USING NEURAL NETWORK APPROACH
}

\author{
MARY JAYA V J \\ Department of Computer Science, Assumption College, Changanacherry
}

\begin{abstract}
The problem of detection and characterization of a dielectric cylinder inside a given investigation domain by means of the scattered field values can be represented in terms of the reconstruction of the dielectric characteristics of a generic body illuminated by an incident electromagnetic wave and by means of scattered field values available at some points in the space outside the investigation domain. A circular Cylinder with unknown radius ' $r$ ', Dielectric permitivity ' $\varepsilon$ ' and center position $(x, y)$ which is illuminated by an incident TM wave produces a scattered electric field which is measured at some point on a circle centered at the axis origin. The unknown dielectric and geometric characteristics of the investigated object can be found by means of a neural network with a single hidden layer. The inputs to the network are the complex values of the scattered field, while the outputs are the unknown variables. The data inputs are trained using the two-layer perceptron training algorithm, the backpropogation algorithm. This algorithm is very slow, and so another version of BP algorithm, called Vogl's acceleration, which allows a fast and more precise convergence is proposed. But when dealing with larger dimension networks, the YPROP algorithm gives a further modification of the BP original procedure by allowing the acceleration and deceleration factors to change at each iteration. After the training step, the neural network is tested with many, differently taken, data. Finally, test results for networks devoted to the retrieval of the dielectric characteristics, or the position, or both. In this work I'm trying to present the application of a neural network approach to the electromagnetic inverse scattering problem and also trying to show that a suitable network could be able to give a sufficiently accurate solution to the problem of retrieving the position, radius, and dielectric permitivity of an unknown cylinder illuminated by a TM wave, starting from the knowledge of the scattered field. The stability and accuracy of the results are to be studied, with particular stress on the possibility to build specialized networks devoted to the separate extraction of the dielectric and geometric characteristics of the scatterer.
\end{abstract}

KEYWORDS: Neural Network, MBP \& YPROP

Received: Aug 20, 2017; Accepted: Sep 17, 2017; Published: Oct 13, 2017; Paper Id.: IJCSEITROCT20174

\section{INTRODUCTION}

Retrieval of shape, size and internal property of objects buried underground using electromagnetic scattering has gained interest in many areas like biomedical engineering etc. [1]. Solution to these problems are inherently ill posed and non-unique and requires time-consuming iterative algorithms. Thus, these techniques are computationally intensive and not suitable for real-time applications. An interesting alternative to these methods are neural networks. Recently, neural-network-based methodologies have been applied to electromagnetic inverse problems. The neural-network approach to the solution of an electromagnetic inverse scattering problem has generally been based on Multilayer Perceptron (MLP) and Radial-Basis Function Neural Network (RBF-NN) [2].

MLP is not suitable for certain application such as online inverse scattering problems. The backpropagation algorithm, which is the training process of an MLP neural network, includes forward and backward propagation with desired output used to generate error values for back propagation in order to improve the output 
iteratively. The back-propagation algorithm is limited by its slow training performance. In contrast, the parameters of the RBF-NN are computed in a straightforward manner. A refined version of the back propagation algorithm, allowing a faster and more precise convergence, is proposed and adopted in training step. The so-called Vogl's acceleration consists of a more clever choice of the weight-updating rule. The learning step is in fact updated at each iteration by means of acceleration or a deceleration factor. The heuristic under this choice is that, if the error decreases (the direction is correct), the learning step can be safely increased. Otherwise, the right direction has been probably lost and a smaller step might be necessary. When dealing with larger dimension networks, the YPROP algorithm gives a further modification of the back propagation original procedure, by allowing the acceleration and deceleration factors (fixed in the Vogl's acceleration algorithm) to change at each iteration. These factors are chosen to let the learning step increase quickly if it is small, but less quickly when it is already large.

\section{Back Propagation Network}

On behalf of universal approximation theorem, using a single hidden layer of neural network, unknown characteristics of the investigation object may be found, where complex values of the scattered field are fed as the input, and the four unknown variables are obtained as the output. It directly gives an output to the given input, once the neural network has been instructed and trained for a certain problem. Thus, neural network is an ethereal solution for online inverse scattering which demands rapid measurement inversions. Decrease in number of input, simplifies the neural-network architecture and reduces the training time.

The final version of the back-propagation algorithm is done as follows:

\section{1) Feed-forward}

for each layer $l:=1$ to $\mathrm{L}$

$$
\begin{aligned}
& \text { for each neuron } n:=1 \text { to } \mathrm{N}_{l} \\
& \text { For each pattern } p:=1 \text { to } \mathrm{P} \\
& s_{n}^{(p)}(l)=f\left(s^{(p)}(l-1)^{T} \cdot w^{(n)}(l)+b_{n}(l)\right)
\end{aligned}
$$

\section{2) Error computation}

for each neuron in the out layer $n:=1$ to $\mathrm{N}_{\mathrm{L}}$

for each pattern $\mathrm{p}:=1$ to $\mathrm{P}$

$\delta_{n}^{(p)}(L)=\frac{2}{P . N_{L}}\left(t_{n}^{(p)}-s_{n}^{(p)}(L)\right)\left(1-\left[s_{n}^{(p)}(L)\right]^{2}\right)$

\section{3) Error back-propagation}

for each layer $l:=\mathrm{L}-1$ to 1

for each neuron $n:=1$ to $\mathrm{N}_{1}$

for each pattern $p:=1$ to $\mathrm{P}$ 


$$
\delta_{n}^{(p)}(l)=\left(1-\left[s_{n}^{(p)}(l)\right]^{2}\right) \cdot \sum_{j=1}^{N_{l+1}}\left[\delta_{j}^{(p)}(l+1) \cdot w_{n}^{(j)}(l+1)\right]
$$

\section{4) Step computation}

for each layer $l:=1$ to $\mathrm{L}$

$$
\text { for each neuron } n:=1 \text { to } \mathrm{N}_{l}
$$

$$
\Delta b_{n}(l)=\eta \sum_{p=1}^{p} \delta_{n}^{(p)}(l)
$$

for each weight $i:=1$ to $\mathrm{N}_{l-1}$

$$
\Delta w_{i}^{(n)}(l)=\eta \sum_{p=1}^{p} \delta_{n}^{(p)}(l) . s_{i}^{(p)}(l-1)
$$

\section{5) Weight updating}

for each layer $l:=1$ to $\mathrm{L}$

for each neuron $n:=1$ to $N_{l}$

$$
b_{n}^{\text {new }}(l)=b_{n}^{\text {old }}(l)+\Delta b_{n}(l)
$$

for each weight $i:=1$ to $N_{l-1}$

$$
w_{i}^{(n), n e w}(l)=w_{i}^{(n), o l d}(l)+\Delta w_{i}^{(n)}(l)
$$

\section{Problems with conventional Back propagation Algorithm}

The back-propagation algorithm described above is terribly slow and can even fail to lower the error E [3]. There are at least two reasons for this poor performance:

- A step in the opposite direction of the gradient can increase the error if it is too big.

- The gradient does not point towards the minimum of the error surface causing an undesirable zigzag effect.

The second problem can be easily solved at least in part using Vogl's acceleration technique. The actual algorithm for gradient descent weight adjustment is basically the learning rate multiplied by the partial derivative of the Mean Square Error (MSE) with respect to the weight value. When gradient descent encounters a MSE versus weight value graph, and if the graph's slope is fairly shallow, and the derivative therefore small, the calculation will yield a small weight change. This can cause many unnecessary weight changes. This situation can cause greater problems when the algorithm encounters a steep surface or trench in the error surface. Since the rate of change is large, the weight change will be proportionally large. This may cause the learning algorithm to overstep the desired answer. These two problems can have troublesome effects on training time and accuracy.

The training time of a back propagation networks tend to be long; this has lead to modify the basic algorithm to adjust the learning parameter and the momentum parameter. So a refined version of the back propagation algorithm, allowing a faster and more precise convergence, called Vogls acceleration consisting of a more clever choice of the weightupdating 


\section{Vogl Acceleration Technique}

The main point of this acceleration technique is to vary the learning step and the momentum during the learning in order to adapt them on-line to the shape of the error surface [4]. The Vogl technique can be briefly summarized as follows:

- $\quad \eta(o)=\eta_{0} ; \alpha(0)=\alpha_{0}$

- If $\mathrm{E}(\mathrm{t})<\mathrm{E}(\mathrm{t}-1)$ then $\eta(t)=\phi . \eta(t-1) ; \alpha(t)=\alpha_{0}$

- If $\mathrm{E}(\mathrm{t})<(1+\varepsilon) E(t-1)$ then $\eta(t)=\beta . \eta(t-1) ; \alpha(t)=0$

- if $\mathrm{E}(\mathrm{t})>(1+\mathcal{E}) \mathrm{E}(\mathrm{t}-1)$ then discard the last step; $\eta(t)=\beta . \eta(t-1) ; \alpha(t)=0$

Where $\mathrm{t}$ is the iteration counter $\phi>1$ is the acceleration factor, $\beta<1$ is the deceleration factor, and $0.01 \leq \varepsilon \leq$ 0.05. The behavior of this technique is straightforward. If the error decreases, the learning step is increased by a factor $\phi$ and the momentum is retained. Otherwise, if the step produces an error greater than the previous value, the learning rate is decreased by a factor $\beta$ and the momentum is discarded. If the error increased only a few percent, the step is accepted. If the initial step is too large, some iteration is wasted at the beginning of the learning until a good $\eta$ is found. On the other hand, this technique is quite sensitive to the acceleration and deceleration parameters as they can heavily influence the convergence speed. Vogl proposes $\phi=1.05$ and $\beta=0.7$.

\section{YPROP: ENHANCED VOGL TECHNIQUE (EVT)}

Instead of setting the acceleration and deceleration factors to fixed values, they are changed them at every learning step using [5]:

$$
\phi(t)=1+\frac{K_{a}}{K_{a}+\eta(t-1)} \quad \text { and } \quad \beta(t)=\frac{K_{d}}{K_{d}+\eta(t-1)} \quad \rightarrow
$$

Where $\mathrm{K}_{\mathrm{a}}$ and $\mathrm{K}_{\mathrm{d}}$ are two constants (respectively for pattern learning acceleration and deceleration). YPROP has the same number of parameters as the original Vogl's. The behavior of the algorithm is the following:

In the acceleration phase, if $\eta(t-1)$ is small $\left(\eta(t-1)<<\mathrm{K}_{\mathrm{a}}\right)$

Then $\eta(t) \sim 2 . \eta(t-1)$, if instead $\eta(t-1)<<\mathrm{K}_{\mathrm{a}}$ then $\eta(t) \sim \eta(t-1)$. The heuristic behind this is the following: we want $\eta$ to increase rapidly if it is very small, but we want to be cautious if $\eta$ is very large. The deceleration phase shows the opposite behavior, if $\eta(t-1) \gg>K_{d}$ then $\eta(t) \cong K_{d}$ In other words the learning step is immediately shortened.

If $\eta(t-1)<<K_{d}$ then $\eta(t) \cong \eta(t-1)$; we are probably stuck in a local minimum, so letting the step almost unchanged can help to escape from it. Both techniques described are based on some heuristic in order to make the descent on the error surface: there are cases in which they can be totally useless.

\section{Advantage of YPROP Algorithm Over Conventional BP Algorithm}

In order to reduce the training time of back propagation in networks, the basic algorithm is modified to adjust the learning parameter $\eta$ and the momentum parameter $\alpha$. For this adjustment, Vogls accelerating technique and the YPROP Algorithm 
are employed. The main point of Vogl's acceleration technique is to vary the learning step and the momentum during the learning in order to adapt them on-line to the shape of the error surface. YPROP is an enhanced version of Vogl acceleration technique. The idea behind this technique is very simple, Instead of setting the acceleration and deceleration factors to fixed values, they are changed at every learning step.

\section{Neural Network Inverse Scattering Approach}

A cylindrical object of arbitrary cross section surrounded by a homogenous exterior medium of known complex permittivity under TM harmonic excitation is located in a rectangular imaging domain I. A cylindrical geometry for the imaging system is employed, with $\mathrm{M}$ line sources equi-spaced on a circle in the measurement domain. At a given time, one of the antennas will be emitting and others will be receiving. The scattered field is measured for different views. A time convention of $\mathrm{e}^{j \omega t}$ is employed [6]. The total field satisfies the scalar electric-field integral equation for the view $\mathrm{v}$ :

$$
\begin{aligned}
& e_{v}(r)=e_{v}^{i n c}(r)+e_{v}^{s c a t}(r) \quad \rightarrow \\
& \text { Where } \\
& e_{v}^{i n c}(r) \text { is the incident field and } e_{v}^{s c a t}(r) \text { is the scattered field, given by } \\
& e_{v}^{\text {scat }}(r)=\iint_{I} k_{0}^{2} c\left(r^{\prime}\right) e_{v}\left(r^{\prime}\right) G\left(r, r^{\prime}\right) d r^{\prime}, v=1,2, \ldots \ldots \ldots \ldots \ldots \ldots . . M \quad \rightarrow
\end{aligned}
$$

where, $\mathrm{G}$ is the 2D free-space Green's function and c the object contrast, given by

$$
c(r)=\in(r)-\epsilon_{e x t} \quad \rightarrow
$$

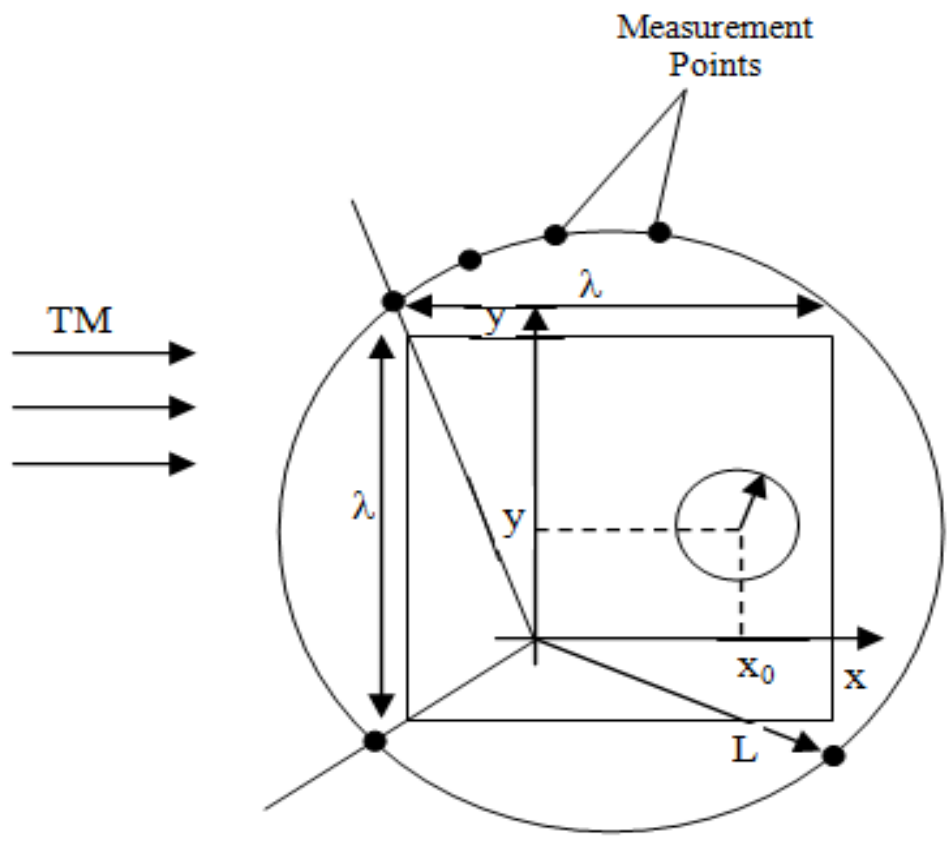

The physical system under investigation: a circular cylinder with unknown radius $r$, dielectric permittivity $\varepsilon_{r}$, and center position $(x ; y)$ is illuminated by an incident $T M$ wave, and the scattered electric field is measured at some point on a circle centered at the axis origin.

$\mathrm{k}_{0}$ is the free space propagation and $\mathrm{k}_{\mathrm{ext}}$ is the propagation constant in the homogenous medium [7]. 
We cope with the nonexistence of the inverse solution by redefining the solution as a minimizer of the leastsquare error with respect to the data. In practice one looks for a spatially discretized vector $c(r)$, the lexicographically ordered representation of the 2D image contrast. This alleviates non-uniqueness to a good extent. This is evident that the stability of the solution will be better when the discretization cell size is not too small [8]. Hence, non-uniqueness is less prominent at a coarse resolution. However, the use of a cost functional may again introduce local minima. The integral equations are discretized. The imaging region is discretized into $N$ cells of dimensions corresponding to the particular scale. The discretized equations are given by

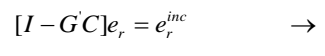

and

$e_{v}^{\text {scat }}=G_{v}^{\text {scat }} E_{v} C \quad \rightarrow$

Where $G_{r}^{\text {inv }}$ and $G_{v}^{\text {scat }}$ are the integrated Green's function matrices of dimensions $N$ x $N$ and $(M-1)$ x $N$, where $(M-1)$ is the number of receivers per view, $C$ and $E_{v}$. are diagonal matrices with $[\mathrm{C}]_{k k}=c[k]$ and $\left[E_{v}\right]_{k k}=\mathrm{e}_{v}[k]$, where $c$ and $e_{v}$ are the discretized lexicographically ordered contrast function and the total electric field in $I$ for the view $v$, respectively.

\section{Two Layer Perceptron}

A two-layer feed forward perceptron network with a nonconstant, bounded, and monotone increasing activation function is sufficient to approximate any nonlinear function $\mathrm{Y}=\mathrm{f}(\mathrm{X})$ relating the two sets of variables $\mathrm{X}$ and $\mathrm{Y}$ which is so called the universal approximation theorem. The physical system to be analyzed is presented in fig. where transverse magnetic (TM) plane waves illuminate a square investigation domain holding eventually a circular cylindric dielectric body with location, radius, and dielectric characteristics that are unknown quantities [9].

This system defines a very complex nonlinear problem, however, if we exploit the a priori information that is in the investigation domain, only circular cylindric objects can be present. In this way, the unknown variables can be reduced. In particular, they become the coordinates of the location of the section center in the xy plane, its radius, and its dielectric permittivity. Magnetic permittivity is considered to be equal to one. On behalf of the universal approximation theorem, we can find the unknown dielectric and geometric characteristics of the investigated object by means of a neural network with a single hidden layer, the inputs are the complex values of the scattered field, while the outputs are the four unknown variables.

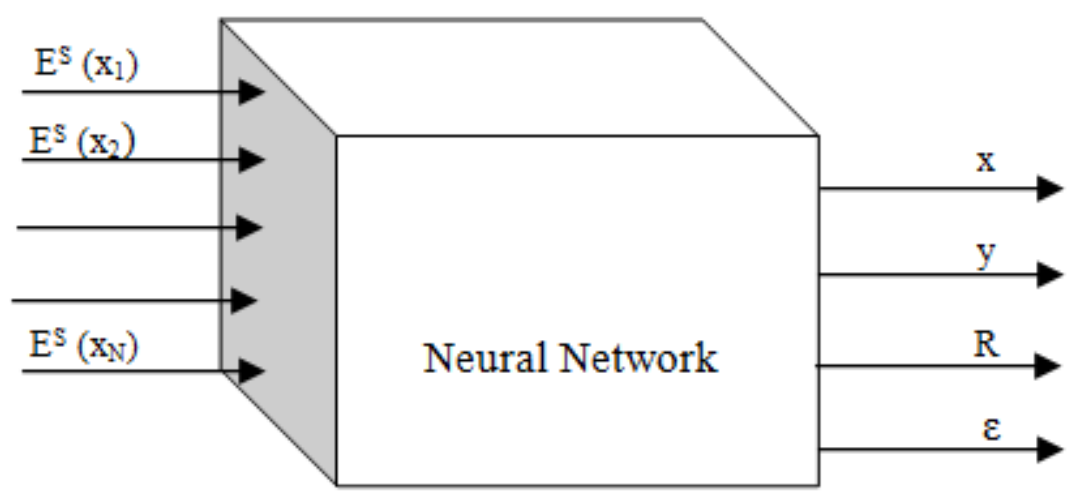

Input and output values of the neural network 
The resulting neural network is as shown in the figure 4. The inputs of the network are given by complex values of the scattered field evaluated in $\mathrm{N}$ points located along an observation line. The outputs are given by the location ( $\mathrm{x}$ and $\mathrm{y})$, the radius $(\mathrm{R})$ and dielectric permittivity $\left(\varepsilon_{\mathrm{r}}\right)$ of the cylinder.

During the training phase, the outputs of the neural network are represented by the well known characteristics of the dielectric object that produced the scattered field applied at the input. Then, once the neural network has been trained, it will be able to provide at its output the unknown characteristics of the object whose scattered field values are applied at the input. The approach used to train the neural network is the YPROP Algorithm, which is another accelerating technique for the Back propagation.

\section{Training Data Sets}

The data sets used for training and testing the network were obtained by a very simple forward model. In fact, for TM plane wave illumination and circular cylinders with known location, radius, and dielectric the scattered electric field can be obtained in a closed form. This model provides the scattered field in a number of points along an arbitrary curve in the xy plane, which, in this case, was chosen as a circular arc centered around the axis origin and opposite to the direction of the incident wave. In particular, 16 equispaced points were chosen along a $3 / 2 \pi$ circular arc as in Fig.3.

Three different kinds of data sets have been used to train the network.

- The first one containing the scattered field values obtained by considering a cylindrical object with known radius and dielectric characteristics but different location.

- The second one composed by the scattered field values obtained by considering cylindrical target with a fixed location but variable dielectric and geometrical properties.

- Finally, a data set is obtained by considering that every parameter characterizing the object can change arbitrarily.

The great advantage of this analysis is the possibility to test the robustness of the neural network approach, and at the same time, to estimate how different training examples affect the behavior of the trained network.

Since a great number of data are required to train the neural network, direct experimental measurements on a physical scenario cannot be used to form the training set. Therefore the development of an appropriate simulator that solves the direct scattering problem providing the values of the scattered field is necessary.

\section{Training Algorithm}

The usual approach to two-layer perceptron training is the back propagation (BP) algorithm. Initially, the weights that define each connection between neurons are randomly chosen. Then, a forward-propagation step computes the output of the network for a given input pattern (the training data) [10]. By a comparison of the obtained output with the desired one, a suitable indication to update the guessed weight is extracted. By iterating the procedure until convergence, the method moves toward a minimum of the rmse. However, usually the algorithm is terribly slow and may even fail to lower the rmse. This is due to the critical choice of the learning step in the gradient-descent algorithm

To solve these problems, a refined version of the BP algorithm, allowing a faster and more precise convergence, which is proposed adopted in the training step. The so-called Vogl's acceleration consists of a more clever choice of the weight-updating rule. The learning step is in fact updated at each iteration by means of acceleration or a deceleration 
factor. The heuristic under this choice is that, if the error decreases, the learning step can be safely increased, otherwise, the right direction has been probably lost and a smaller step might be necessary. It is also found useful that, when dealing with larger dimension networks, the YPROP algorithm gives a further modification of the BP original procedure by allowing the acceleration and deceleration factors (fixed in the Vogl's acceleration algorithm) to change at each iteration. These factors are chosen to let the learning step increase quickly if it is small, but less quickly when it is already large. In other words, the learning step is no more a linear function of the gradient of the rmse.

The detection and characterization of an object inside a given investigation domain by means of the scattered field values can be represented in terms of the reconstruction of the dielectric characteristics of a generic body illuminated by an incident electromagnetic wave and by means of scattered field values available at some points in the space outside the investigation domain.

\section{Generation of Test Data}

Here the object used is a circular cylinder with unknown radius ' $r$ ', Dielectric permittivity ' $\varepsilon_{r}$ ' and center position ( $\left.x, y\right)$. This object is illuminated by a transmitting antenna, which is kept at an angle $\pi / 2$ with respect to the plane of the object [9]. The incident TM wave produces a scattered electric field, which is measured at some point on a circle centered at the axis origin. This scattered electric field is measured using 16 receiving antennas placed at different angles with respect to the axis of the object. The receiving angle is calculated using

Receiver angle $=\pi / 4+(\mathrm{rr}-1) * \mathrm{pi} /(2 *(\operatorname{rmax}-1))$

where rr varies from 1 to 16 and rmax is the maximum number of receivers used in the arrangement. Thus the antennas are arranged at angles extending from $\pi / 4$ to $-\pi / 4$.

Next is to generate the profile of the object. It is computed using the coordinates of the object points, the profile of the object contrast, permittivity and its vector representation, which is lexicographically ordered. The object contrast depends on the permittivity of the object and the medium. The incident field from the linesource-transmitting antenna is calculated using the transmitter coordinates and the object coordinates. Next the greens function and the inverse, matrix are employed to compute he total field inside the object given its permittivity. The inverse green function is used to generate the test data, which gives the measure of the scattered field from the object. Different set of test datas are generated by varying the radius, position (i.e, varying the $\mathrm{x}$ and $\mathrm{y}$ coordinates) and permittivity of the object. The test data is then fed to a neural network for the efficient generation of the unknown parameters of the circular cylindrical object.

\section{RESULTS AND DISCUSSION}

The basic method utilizes design knowledge based on material properties and geometric properties of the cylindrical object that can even remotely influence the parameters of the object. Design parameters like radius of the cylindrical object, its dielectric characteristics, and its location are measured at different span locations, and used as initial variables. The net is constructed. All the charecters are normalized and the net is trained in a normalized design space. In order to reduce the training time of backpropagation in networks, the basic algorithm is modified to adjust the learning parameter $\eta$ and the momentum parameter $\alpha$. For this adjustment, Vogls accelerating technique and the YPROP Algorithm are employed. An electric line source is supposed to illuminate a square domain. The relative dielectric permittivity of the unknown cylinder is changed into a range of values of $[1 ; 5]$ while the radius can vary in a range of $[0.2 ; 0.5]$ normalized with respect to the 
domain side, and the position coordinates changed to a range of $[-2.5 ;+2.5]$. Sixteen observation points have been taken into account to evaluate the scattered field used to form the training/test data. About 500 sets of data were generated by varying the radius, permittivity and position of the unknown cylinder.

In the analysis of the efficiency of the neural network approach in the reconstruction of a cylinder located in a homogenous domain, architecture with four outputs have been implemented. In this case, the neural network showed a satisfactory behavior. The result seems to show that the location and the dielectric/geometric characteristics of the unknown cylinder can be considered as uncorrelated parameters for a well pre-defined investigation domain. The neural network response with four outputs i.e., position (x,y), radius $\mathrm{R}$ and permittivity $\varepsilon_{\mathrm{r}}$ is as shown below

- $\quad$ Result obtained after training 121 sets of data which are obtained by varying the $\mathrm{x}$ and $\mathrm{y}$ coordinates keeping the radius and permittivity constant.

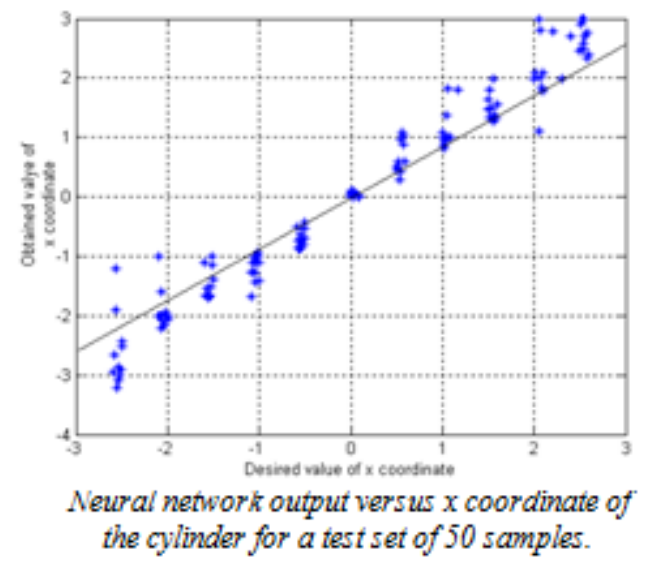

Good results have been obtained for a neural network with only two outputs i.e., the $\mathrm{x}$ and $\mathrm{y}$ coordinates of a given cylinder. The distribution of the $\mathrm{x}$ and $\mathrm{y}$ coordinates are cluttered around the origin. In this case the reconstructed values are quite similar to real ones.

- $\quad$ Result obtained after training 12 sets of data which are obtained by varying the permittivity alone keeping the $\mathrm{x}$ and $\mathrm{y}$ coordinates as well as radius constant

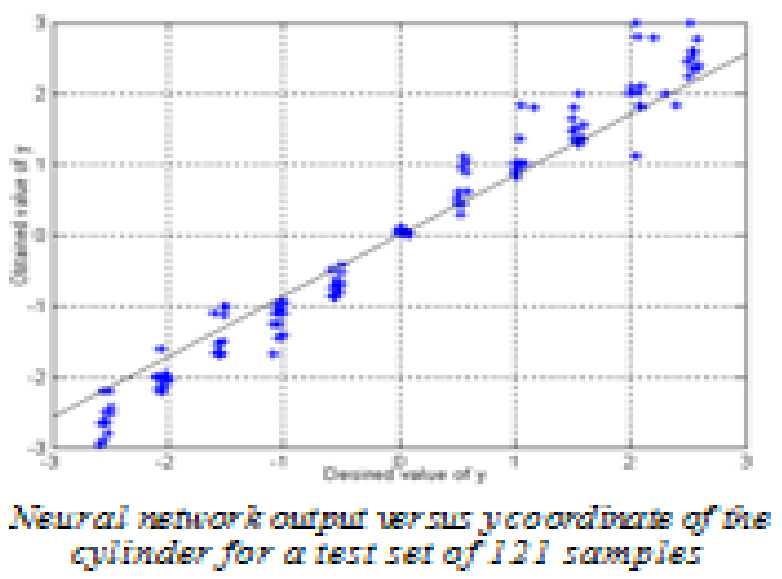


- $\quad$ Result obtained after training 10 sets of data which are obtained by varying the radius alone keeping the $\mathrm{x}$ and y coordinates as well as permittivity constant.
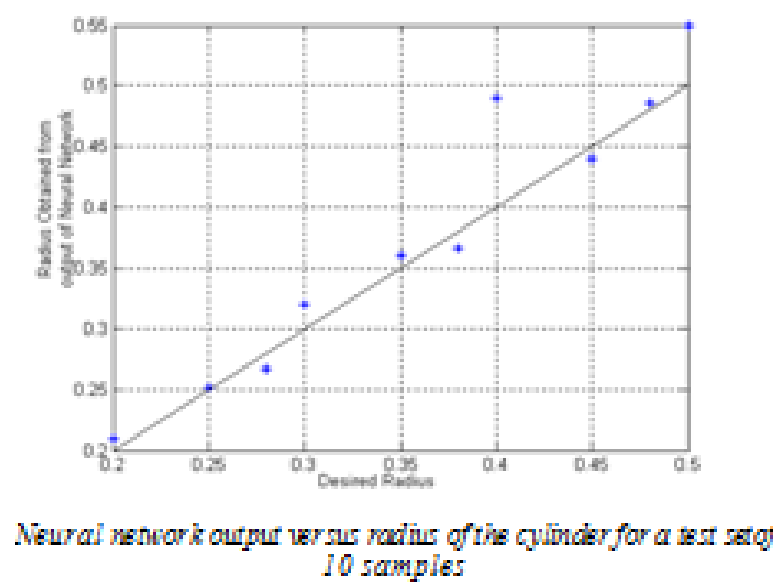

10 samples

- $\quad$ The output of a neural network with four outputs obtained by varying all the four parameters.

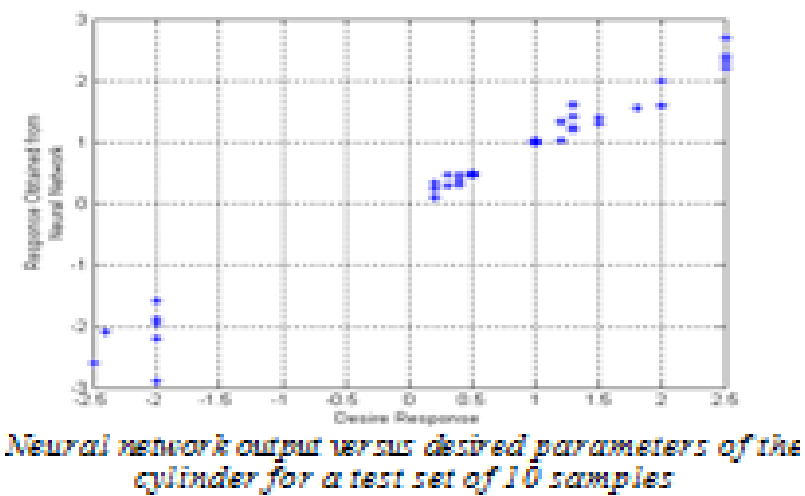

\section{CONCLUSIONS}

In this work, the application of a neural network approach to the electromagnetic inverse scattering problem is presented. It is shown that a suitable network could be able to give a sufficiently accurate solution to the problem of retrieving the position, radius, and dielectric permittivity of an unknown circular cylinder illuminated by a TM wave, starting from the knowledge of the scattered electric field. The stability and accuracy of the results has been investigated, with particular stress on the possibility to build specialized networks devoted to the separate extraction of the dielectric and geometric characteristics of the scatterer. The results are encouraging, and they show that the application of a neural approach to the electromagnetic inverse scattering is valid and that more research should be done to widen this extremely interesting method to more general situations.

A circular cylindrical object with unknown radius ' $\mathrm{r}$ ', dielectric permittivity ' $\varepsilon_{\mathrm{r}}$ ' and center position ( $\mathrm{x}$, y), illuminated by a transmitting antenna, was kept at an angle $\pi / 2$ with respect to the plane of the object. The incident TM wave produced a scattered electric field, which was measured at some point on a circle centered at the origin. The scattered electric field was measured using 16 receiving antennas placed at different angles with respect to the axis of the object. About 500 sets of data were generated. 
A network with forty-eight nodes at the hidden layer was constructed. When different sets of data were passed through the network a response similar to the desired response was obtained. After training and fixing the synaptic weights the neural network acted as a black box giving the necessary desired responses, whatsoever data is applied to the input of the network. When scattered electric field data, obtained by illuminating a circular object using TM waves, were passed through this network, the corresponding outputs obtained at the output of the network are the required parameters of the object such as position, radius and dielectric permittivity of the circular cylindrical object. The preliminary results show that the method is extremely flexible and very suitable for data analysis on field.

\section{REFERENCES}

1. Benedetti, Manuel, et al. "An innovative microwave-imaging technique for nondestructive evaluation: applications to civil structures monitoring and biological bodies inspection." IEEE Transactions on Instrumentation and Measurement 55.6 (2006): 1878-1884.

2. Electromagnetic Detection of Dielectric Cylinders by a Neural Network Approach, Salvatore Caorsi and Paolo Gamba, IEEE Transactions on Geoscience and Remote Sensing, Vol. 37, No.2, March 1999.

3. D.E Rumelhart, G.E. Hinton, and R.H Williams, "Learning representations by back propagation errors," Nature, vol. 323,pp.533-536, 1986.

4. T. P. Vogl, J. K. Mangis, A. K. Rigler, W. T. Zink, and D. L. Alkon, “Accelerating the convergence of the back-propagation method," Biol. Cybern., vol. 59, pp. 257-263.

5. D. Anguita, M. Pampolini, G. Parodi, and R. Zunino, "YPROP: Yet another accelerating technique for the back propagation," in Proc. Int. Conf. Artif. Neural Networks, Amsterdam, The Netherlands, Sept. 13-16, 1993, pp. 500-503.

6. H.Hidalgo and E. Gomez- Trevino," Application of constructive learning algorithms to the inverse problem, " IEEE Trans. On Geoscience and Remote Sensing, vol.34, no.4, pp. 874-885, July 1996.

7. Microwave Imaging Of 2 D Dielectric Cylinders With A Multiscaled Frequency Hopping approach, Microwave And Optical Technology Letters / Vol. 43, No.4, November 202004.

8. I.T. Rekanos, Neural-network-based inverse-scattering technique for online microwave medical imaging, IEEE Trans Magn 38 (2002), 1061-1064.

9. E.Bermani, S. Coarsi, and M. Raffetto, Microwave detection and dielectric characterization of cylindrical objects from amplitude-only data by means of neural networks, IEEE Trans Antennas Propagat 50 (2002), 1309-1314.

10. S. Haykin, Neural Networks, New York: Macmillan, 1994. 
